\title{
Variables físicas que explican la variabilidad de suelo aluvial y su comportamiento espacial
}

\author{
Daniel Francisco Jaramillo Jaramillo(1), María Luisa Anaya Gómez ${ }^{(1)}$, Carlos Andrés Restrepo Moná(1), \\ Hugo Alberto González Sánchez ${ }^{(1)}$ y Fernando Álvarez Mejía ${ }^{(1)}$ \\ (1)Universidad Nacional de Colombia, Sede Medellín, Calle 59 A, no 63-20, Medellín, Colombia. E-mail: djaramal@unal.edu.co, \\ mlanaya@unal.edu.co, carestr2@unal.edu.co, hagonzal@unal.edu.co, falvarez@unal.edu.co.
}

Resumen - El objetivo de este trabajo fue utilizar el análisis de componentes principales y de semivarianza para seleccionar variables físicas que explicaran la variabilidad de un suelo aluvial, y establecer el comportamiento espacial de las variables seleccionadas, con el fin de definir técnicamente la localización de parcelas experimentales para estudiar los efectos de la abrasividad del suelo sobre el desgaste de herramientas agrícolas. Las pruebas de campo se realizaron en 2008, en un lote plano de $6.000 \mathrm{~m}^{2}$ con suelos de textura media a pesada (Vertic Haplustepts). Se hizo un muestreo intensivo en cuadrícula de 10x14 m. Las variables que mayor peso tuvieron en los tres primeros componentes principales fueron los contenidos de limo, arena fina y media, gravilla media, la humedad a capacidad de campo y el coeficiente higroscópico. A excepción de la arena media y la capacidad de campo, las demás propiedades presentaron alta dependencia espacial y su distribución mostró que en el lote experimental se encuentran tres sectores de acumulación diferencial de limo y de arena fina. La combinación de los análisis de componentes principales y geoestadística permitió definir las propiedades del suelo involucradas en el desgaste de herramientas, su patrón espacial y la manera más adecuada de distribuir parcelas experimentales, para estudiar la abrasividad del suelo.

Términos para indexación: abrasividad, análisis de tendencia, componentes principales, dependencia espacial, geoestadística, textura del suelo.

\section{Physical variables that explain alluvial soil variability and their spatial behavior}

\begin{abstract}
The objective of this work was to use the principal component and semivariance analyses to select physical variables that could explain the variability of an alluvial soil, in order to establish the spatial behavior of the chosen variables so that the location of experimental plots could be technically defined to study the abrasiveness effect on the wearing away of farm equipment. Field tests were performed in 2008, on a $6,000 \mathrm{~m}^{2}$ flat lot with medium to heavy soil texture (Vertic Haplustepts). An intensive sampling was done in a grid of 10x14 m. The variables which had the most weight on the first three principal components were the contents of silt, fine and intermediate sand, intermediate gravel, soil moisture at field capacity, and the hygroscopic coefficient. Except for the half sand and the field capacity, the other properties showed a high spatial dependence, and their distribution showed that in the experimental plot there are three sectors of differential accumulation of silt and fine sand. The combination of principal component analysis and geostatistics allowed for defining the soil properties involved in the wear of tools, their spatial pattern and the most appropriate way of distributing plots to study soil abrasiveness.
\end{abstract}

Index terms: abrasiveness, tendency analysis, principal components, spatial dependence, geostatistics, soil texture.

\section{Introducción}

Cuando se quiere estudiar el efecto del suelo sobre el desgaste de los implementos agrícolas, se encuentran muchas propiedades de éste que pueden intervenir $\mathrm{y}$, además, que la mayoría de ellas tienen una alta variabilidad, tanto aleatoria como espacial (Arango, 2004; Lozano et al., 2004; Iqbal et al., 2005; Zeleke \&
Zi, 2005; Zhao et al., 2007; Jaramillo Jaramillo et al., 2008; Souza et al., 2009; Peña et al., 2009).

La posibilidad de encontrar alta variabilidad en las propiedades físicas y mecánicas del suelo implica que se debe tener especial cuidado en la selección de los sitios y en la ubicación de las parcelas experimentales, pues la localización de éstas puede tener efectos sobre los resultados de los análisis de varianza que

Pesq. agropec. bras., Brasília, v.46, n.12, p.1707-1715, dez. 2011 
se hagan, ya que el error experimental puede tener algún componente espacial que afecte la significancia de los tratamientos que se estudian. Este aspecto fue demostrado en algunas investigaciones (Bhatti et al., 1991; Schabenberger \& Pierce, 2002; Jaramillo Jaramillo, 2005, 2006) que evidenciaron que si no se neutraliza la correlación espacial entre unidades experimentales vecinas, se pueden invalidar los resultados obtenidos con el análisis de varianza entre ellas.

El análisis de semivarianza puede ayudar en la selección y ubicación de los sitios experimentales debido a que una de sus aplicaciones más útiles es producir isomapas de las propiedades que se estudian (Webster \& Oliver, 2007; Hengl, 2009). Se debe hacer una zonificación del terreno para separar áreas relativamente homogéneas en el comportamiento de las propiedades del suelo relacionadas con el fenómeno que se quiere dilucidar, de modo que se puedan distribuir las unidades experimentales bajo condiciones comparables y manejables, labor en la que son fundamentales el semivariograma y el interpolador kriging (Lozano P. et al., 2004).

Cuando una determinada cualidad del suelo se relaciona con muchas de sus propiedades, se deben seleccionar aquellas más relevantes. Técnicas geoestadísticas multivariadas pueden de suministrar medidas cuantitativas de interacciones complejas entre propiedades del suelo, y son particularmente útiles para formular hipótesis sobre qué causa específicamente la variabilidad (Bourennane et al., 2004).

Se han utilizado diferentes técnicas para agrupar variables que permitan estudiar el comportamiento espacial de atributos del suelo y de la producción agropecuaria. Castañeda et al. (2010) utilizaron regresión múltiple para establecer cuáles propiedades del suelo, de entre un conjunto de 35 , se relacionaban significativamente con la producción de banano y establecer si era posible incluirlas en un programa de manejo específico por sitio para ese cultivo en la región de Urabá, Colombia. Zaman \& Schumann (2006) también utilizaron regresiones para seleccionar las propiedades del suelo y los contenidos foliares de nutrientes que se relacionaron con la producción de cítricos, para luego establecer áreas homogéneas de manejo de nutrición con métodos geoestadísticos.

Peña et al. (2009) usaron análisis de conglomerados para agrupar propiedades del suelo que luego, con análisis de varianza anidados y semivariogramas, les permitió definir la mejor distancia de muestreo del suelo para maximizar la eficiencia y rentabilidad de esta práctica.

Guastaferro et al. (2010) utilizaron el análisis de componentes principales para reducir el número de variables a tener en cuenta para definir zonas de manejo en programas de agricultura de precisión. Chen et al. (2004) utilizaron el análisis de componentes principales para derivar información específica de fotografías aéreas a color que les permitió eliminar la interferencia que producía la materia orgánica en el color de la superficie del suelo desnudo y pudieron hacer un mapa de la distribución superficial de arcilla en un campo de 115 ha.

Van Meirvenne (2003), mediante el análisis de componentes principales, pudo seleccionar los contenidos de arena, limo y materia orgánica como las variables que condicionaron el manejo del suelo en un programa de agricultura de precisión en campos agrícolas de Bélgica.

Demey et al. (1994) clasificaron 529 fincas arroceras de Venezuela mediante el uso del análisis de componentes principales. Partiendo de un conjunto de 41 variables técnicas, socioeconómicas, agroecológicas e institucionales, llegaron a definir cuatro tipos de finca con base en sólo cinco variables. Hengl (2009) apunta que el análisis de componentes principales es una técnica útil para eliminar información redundante, por lo que ayuda eficientemente a determinar el subgrupo más pequeño de predictores significativos.

El objetivo de este estudio fue realizar un análisis de componentes principales y de semivarianza para seleccionar las propiedades físicas que más se relacionaron con la variabilidad de un suelo de textura pesada, con el fin de definir técnicamente la ubicación de las parcelas experimentales que servirán para un estudio de desgaste de cinceles en el laboreo del suelo.

\section{Materiales y Métodos}

El estudio se inició en abril de 2008 en el lote № 8 del Centro Agropecuario Cotové, de la Universidad Nacional de Colombia, Sede Medellín (Figura 1A), ubicado en el municipio de Santafé de Antioquia,

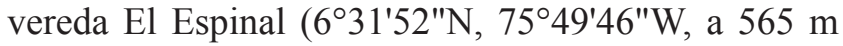
de altitud). Los suelos estudiados han estado sometidos a labranza tradicional por más de 30 años. El Centro Agropecuario se encuentra en un sitio con clima 
cálido seco (bs-T), caracterizado por una temperatura promedia mensual de $27,3{ }^{\circ} \mathrm{C}$, una precipitación promedia anual de $1.019,6 \mathrm{~mm}$ y una evapotranspiración promedia anual de $1.611 \mathrm{~mm}$. El mes más lluvioso
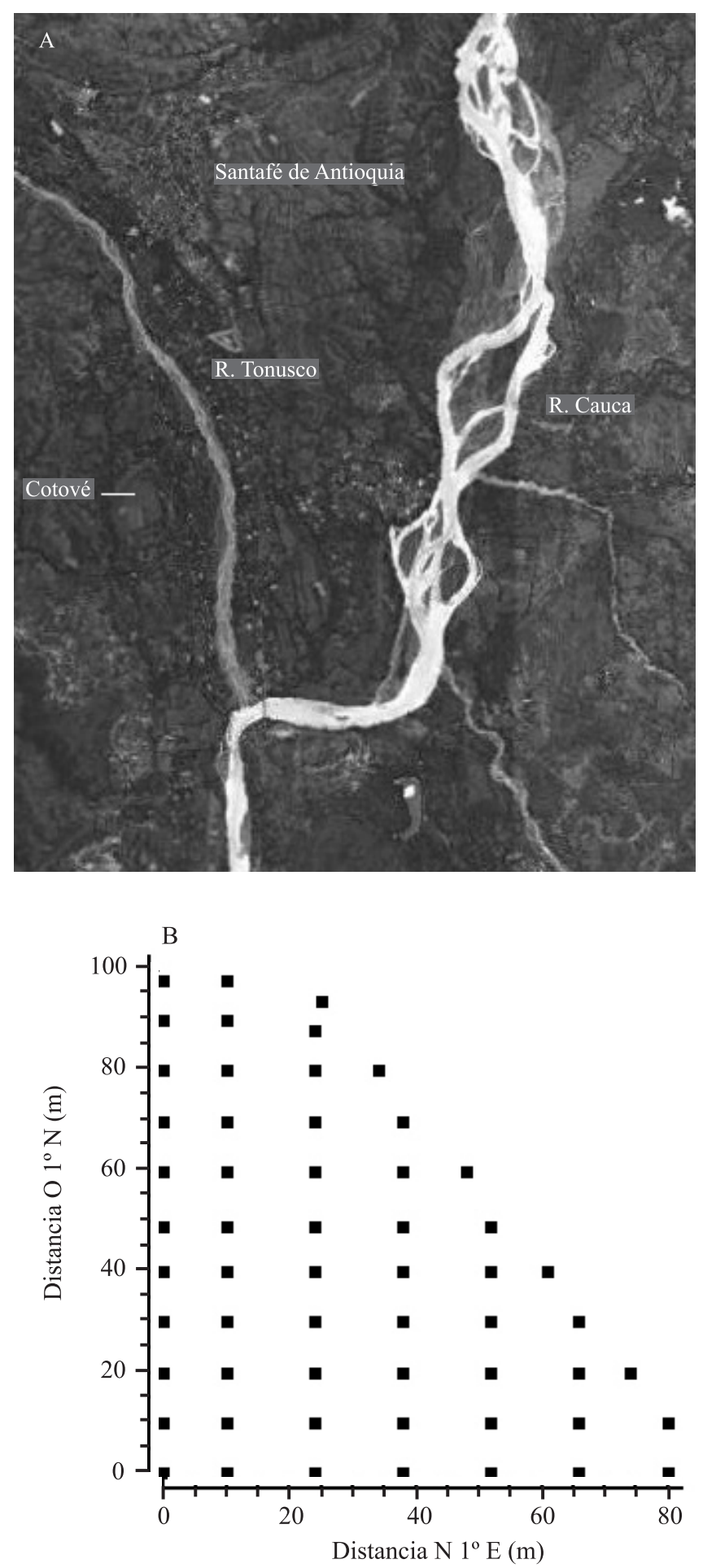

Figura 1. A, localización geográfica del area experimental; $\mathrm{B}$, esquema de muestreo del sitio experimental. es octubre, y el más seco es enero. Se presenta un déficit total de agua promedio anual de 591,4 $\mathrm{mm}$. El lote se ubica en una terraza aluvial plana (pendiente $<3 \%$ ) y los suelos dominantes (Vertic Haplustepts) son de textura media a pesada, moderadamente profundos, de buen nivel de fertilidad (Jaramillo Jaramillo et al., 2008).

En el lote seleccionado, se localizó una parcela de aproximadamente $6.000 \mathrm{~m}^{2}$ en la que se estableció una cuadrícula con tamaño de celda de 14x10 m (Figura 1 B), obteniéndose una malla con 57 intersecciones, que fueron georreferenciadas con un sistema de coordenadas planas $(\mathrm{X}, \mathrm{Y})$ de origen arbitrario. En cada intercepto, se tomó una muestra de suelo sin disturbar con un cilindro biselado de $96,21 \mathrm{~cm}^{3}$ de volumen, para determinar densidad aparente $(\mathrm{Da})$, y otra muestra disturbada entre 15 y $20 \mathrm{~cm}$ de profundidad.

Con la muestra disturbada se determinó la textura del suelo mediante tamizado en húmedo: gravas (Gv: partículas con diámetro entre 75 y $250 \mathrm{~mm}$ ), gravilla gruesa (Gg: partículas con diámetro entre 20 y $75 \mathrm{~mm}$ ), gravilla media $(\mathrm{Gm}$ : partículas con diámetro entre 5 y $20 \mathrm{~mm}$ ), gravilla fina (Gf: partículas con diámetro entre 2 y $5 \mathrm{~mm}$ ), arena muy gruesa (Amg: partículas con diámetro entre 1,19 y $2 \mathrm{~mm}$ ), arena gruesa (Ag: partículas con diámetro entre 0,5 y $1,19 \mathrm{~mm})$, arena media (Am: partículas con diámetro entre 0,25 y $0,5 \mathrm{~mm}$ ), arena fina (Af: partículas con diámetro entre 0,15 y $0,25 \mathrm{~mm}$ ) y arena muy fina (Amf: partículas con diámetro entre 0,05 y $0,15 \mathrm{~mm}$ ). Con el hidrómetro, se determinaron los contenidos de limo (L: partículas con diámetro entre 0,05 y $0,02 \mathrm{~mm}$ ) y de arcilla (Ar: partículas con diámetro $<0,002 \mathrm{~mm}$ ). Se determinaron los contenidos de humedad gravimétricos a capacidad de campo (CC: a $33 \mathrm{kPa}$ ), en el suelo seco al aire (coeficiente higroscópico: $\mathrm{CH}$ ) y en el límite de soltura (LS: humedad donde el suelo empieza a mostrar adhesividad). Se evaluó, además, el contenido de materia orgánica (MO) por el método de Walkley y Black.

Con los resultados obtenidos se hicieron análisis estadísticos exploratorios y luego se realizó un análisis de componentes principales, con las variables estandarizadas, para seleccionar aquellas con mayor peso en los tres primeros componentes principales y dejarlas como variables críticas en la abrasividad del suelo sobre las herramientas de labranza.

A las variables seleccionadas en el análisis de componentes principales se les hizo todo el 
procedimiento de análisis de semivariografía como lo describe Jaramillo Jaramillo (2009) y, finalmente, a aquellas que presentaron dependencia espacial se les hicieron mapas de isolíneas mediante una interpolación con kriging puntual. Con una interpretación visual de los mapas se definieron las áreas más convenientes para ubicar las parcelas experimentales de un futuro experimento, con el fin de evaluar el desgaste de los cinceles en el laboreo del suelo. Todos los análisis estadísticos se hicieron con los programas Statgraphics 5.1 plus y GS+9.0.

\section{Resultados y Discusión}

Las propiedades $\mathrm{CH}, \mathrm{CC}$, LS y Da presentaron coeficientes de variación bajos; en las demás, estos coeficientes fueron altos, llegándose a valores mayores de $150 \%$ en $\mathrm{Gm}$ y Gg, y de $360 \%$ en Gv. La mayoría de las muestras presentaron más de $30 \%$ de Ar y de L, $\mathrm{y}$ contenidos bajos en todas las fracciones de arena. La mayoría de las muestras presentaron contenido alto de MO y de humedad a CC y en el LS. La Da estuvo en niveles adecuados en la mayoría de las muestras, teniendo en cuenta la textura de los suelos (Cortés Lombada \& Malagón Castro, 1984). Sólo las propiedades Am, Ag, Amg, CH, CC, LS y Da presentaron distribución normal y simétrica, la $\mathrm{MO}$ fue simétrica pero no normal. En más del $75 \%$ de las muestras no se presentaron gravas (Gv) y en el $25 \%$ de ellas no hubo gravillas gruesas (Gg) (Cuadro 1). El alto porcentaje de muestras sin Gv permite descartar esta variable para los análisis siguientes. Resultados similares a los anteriores, sobre todo en la variabilidad, fueron obtenidos por Arango (2004) en otro lote experimental de la misma finca. El comportamiento observado en las propiedades analizadas puede estar influenciado por el hecho de que el lote experimental se encuentra en una terraza aluvial de un rio trenzado, en el cual no hay patrones de sedimentación definidos, y por el tiempo que ha estado sometido a laboreo convencional intensivo.

En el Cuadro 2 se observa que los primeros cinco componentes principales explican aproximadamente el $75 \%$ de la variabilidad de las propiedades estudiadas. El Cuadro 3 muestra los coeficientes de las variables en los tres primeros componentes principales, los cuales explican el $60 \%$ de la variabilidad. Se resaltan los coeficientes de mayor valor (absoluto) que son los de las variables con mayor peso dentro de cada componente.

Con la información del Cuadro 3 y de la Figura 2, se seleccionaron las variables más relevantes en los tres componentes, teniendo en cuenta la magnitud y dirección del vector respectivo, así como su relación con

Cuadro 1. Principales estadísticos ${ }^{(1)}$ de las variables analizadas ${ }^{(2)}$.

\begin{tabular}{|c|c|c|c|c|c|c|c|c|c|c|}
\hline Propriedad & Prom & Med & $\mathrm{DE}$ & C.V. & Min & Máx & Q1 & Q3 & Sesgo & Norm \\
\hline $\mathrm{Ar}$ & 28,80 & 31,82 & 9,39 & 32,58 & 5,74 & 39,85 & 27,86 & 34,84 & $-4,23$ & $2,121 \mathrm{E}-10$ \\
\hline $\mathrm{L}$ & 37,13 & 34,75 & 11,44 & 30,82 & 15,16 & 65,96 & 30,98 & 37,47 & 4,41 & $4,213 \mathrm{E}-11$ \\
\hline Amf & 4,75 & 4,25 & 1,48 & 31,26 & 2,51 & 10,34 & 3,71 & 5,58 & 3,88 & 0,0002633 \\
\hline Af & 5,12 & 4,94 & 1,28 & 24,94 & 2,96 & 9,3 & 4,21 & 5,79 & 2,82 & 0,0165444 \\
\hline Am & 4,84 & 4,91 & 0,95 & 19,51 & 2,62 & 7,29 & 4,29 & 5,43 & 0,16 & 0,973329 \\
\hline $\mathrm{Ag}$ & 8,71 & 8,16 & 2,93 & 33,67 & 2,88 & 14,94 & 6,56 & 10,73 & 0,84 & 0,253861 \\
\hline Amg & 2,66 & 2,6 & 1,23 & 46,30 & 0,24 & 5,75 & 1,64 & 3,42 & 1,69 & 0,131581 \\
\hline Gf & 2,31 & 2,05 & 1,43 & 62,02 & 0,19 & 6,66 & 1,27 & 3,12 & 2,86 & 0,002913 \\
\hline $\mathrm{Gm}$ & 1,95 & 1,09 & 3,15 & 161,52 & 0,1 & 22,75 & 0,58 & 2,7 & 16,58 & 0,0 \\
\hline $\mathrm{Gg}$ & 2,02 & 0,6 & 3,45 & 171,23 & 0,0 & 18,4 & 0,0 & 1,98 & 9,08 & 0,0 \\
\hline $\mathrm{Gv}$ & 1,71 & 0,0 & 6,15 & 360,12 & 0,0 & 35,19 & 0,0 & 0,0 & 13,04 & 0,0 \\
\hline $\mathrm{CH}$ & 6,25 & 6,26 & 0,44 & 7,04 & 5,27 & 7,24 & 6,01 & 6,47 & 0,38 & 0,64172 \\
\hline $\mathrm{CC}$ & 28,31 & 28,14 & 1,66 & 5,86 & 24,72 & 33,25 & 27,38 & 29,31 & 1,29 & 0,52166 \\
\hline $\mathrm{LS}$ & 21,33 & 21,65 & 2,49 & 11,67 & 15,22 & 25,9 & 19,47 & 22,92 & $-0,57$ & 0,47556 \\
\hline MO & 6,14 & 4,8 & 2,74 & 44,68 & 3,0 & 11,4 & 3,8 & 8,8 & 1,47 & $2,9866 \mathrm{E}-8$ \\
\hline $\mathrm{Da}$ & 1,41 & 1,4 & 0,131 & 9,46 & 0,96 & 1,84 & 1,34 & 1,48 & $-0,08$ & 0,49776 \\
\hline
\end{tabular}

${ }^{(1)}$ Med, mediana; DE., desviación estándar; CV, coeficiente de variación (\%); Min, mínimo valor; Máx, máximo valor; Q1, cuartil inferior; Q3, cuartil superior; Sesgo, si sesgo varía entre 2 y -2, la distribución de los datos de la variable es simétrica; Norm, valor p del estadístico de normalidad de Shapiro-Wilk: si Norm $>0.05$, la distribución de los datos de la variable es normal. ${ }^{(2)} \mathrm{Ar}$, arcilla; L, limo; Amf, arena muy fina; Af, arena fina; Am, arena media; Ag, arena gruesa; Amg, arena muy gruesa; Gf, gravilla fina; Gm, gravilla media; Gg, gravilla gruesa; Gv, grava; CH, coeficiente higroscópico; CC, capacidad de campo; LS, límite de soltura; MO, materia orgánica; Da, densidad aparente. 
los vectores cercanos. Las propiedades seleccionadas fueron L, Af, Am, Gm, CC y $\mathrm{CH}$, y se observa que corresponden a las partículas gruesas del suelo y a las condiciones de humedad del mismo, que Natsis et al. (2008) encontraron como responsables del desgaste por abrasividad en vertederas.

Las variables seleccionadas en el análisis de componentes principales se sometieron a análisis de variabilidad espacial para lo cual, previamente, se les confirmaron los supuestos de normalidad y de estacionaridad (Diggle \& Ribeiro Junior, 2007; Webster \& Oliver, 2007; Hengl, 2009; Jaramillo Jaramillo, 2009). Las variables Am, CC y CH presentan distribución normal y simétrica, y L, Af y Gm no cumplieron ninguno de estos requisitos. Se hizo una transformación a log de estas últimas tres variables con la que se consiguió simetría en todas ellas, y además normalidad en la distribución de Af y Gm.

Se hicieron regresiones múltiples con un modelo cuadrático (Schabenberger \& Pierce, 2002; Diggle \& Ribeiro Junior, 2007; Krasilnikov \& Sidorova, 2008; Jaramillo Jaramillo, 2009) entre Am, CC, CH, logAf, $\log \mathrm{Gm}$ y $\log \mathrm{L}$ y las coordenadas de los puntos de muestreo, para estudiar la tendencia espacial en las variables, la cual se presentó en $\log \mathrm{L}, \log \mathrm{Af}$ y $\mathrm{CH}$; las otras variables no mostraron ninguna tendencia espacial. Se confirmaron nuevamente los supuestos de normalidad y/o simetría en los residuales de las variables que tuvieron tendencia, y se encontró que en todas las variables se cumplieron estos supuestos,

Cuadro 2. Valores propios de los componentes principales y varianza explicada por ellos.

\begin{tabular}{lccc}
\hline $\begin{array}{l}\text { Componente } \\
\text { principal }\end{array}$ & $\begin{array}{c}\text { Valor } \\
\text { propio }\end{array}$ & $\begin{array}{c}\text { Porcentaje } \\
\text { de varianza }\end{array}$ & $\begin{array}{c}\text { Porcentaje } \\
\text { acumulativo }\end{array}$ \\
\hline 1 & 468,952 & 31,263 & 31,263 \\
2 & 252,495 & 16,833 & 48,096 \\
3 & 17,973 & 11,982 & 60,078 \\
4 & 115,359 & 7,691 & 67,769 \\
5 & 103,501 & 6,9 & 74,669 \\
6 & 0,889792 & 5,932 & 80,601 \\
7 & 0,759279 & 5,062 & 85,663 \\
8 & 0,604374 & 4,029 & 89,692 \\
9 & 0,502009 & 3,347 & 93,039 \\
10 & 0,374903 & 2,499 & 95,538 \\
11 & 0,241988 & 1,613 & 97,151 \\
12 & 0,159789 & 1,065 & 98,216 \\
13 & 0,124572 & 0,83 & 99,046 \\
14 & 0,0794874 & 0,53 & 99,576 \\
15 & 0,0634286 & 0,423 & 100 \\
\hline
\end{tabular}

además de la estacionaridad. Con este resultado se definieron las variables a utilizar en el análisis de semivariografía: datos originales de Am y de CC, datos transformados a $\log$ de $\mathrm{Gm}(\log \mathrm{Gm})$ y los residuales de $\mathrm{CH}$ (resCH) y de log de Af y log de L (reslogAf y reslogL, respectivamente).

El Cuadro 4 muestra los resultados de los análisis de semivariografía realizados. Am y CC no presentaron dependencia espacial. Las otras variables presentaron dependencia espacial media (reslogL) y alta $(\log \mathrm{Gm}$, resCH y reslogAf), según la clasificación propuesta por Cambardella et al. (1994). Los modelos que mejor ajustaron a los semivariogramas experimentales fueron isotrópicos y en ninguno de ellos se presentó periodicidad que hiciera suponer la presencia de alguna tendencia en los datos (Krasilnikov, 2008) ni variabilidad a otras escalas (Webster \& Oliver, 2007). Excepto en el L, que presentó un rango de $90 \mathrm{~m}$, en las demás variables la dependencia espacial fue de rango corto: entre 12 y $24 \mathrm{~m}$. Ninguna de las variables analizadas presentó un comportamiento similar al observado por Arango (2004) en un sector cercano al de este trabajo.

Para las variables que presentaron dependencia espacial se realizó una interpolación con el método del kriging puntual y se elaboraron sus correspondientes mapas de isolíneas (Figura 3), para conocer el patrón

Cuadro 3. Peso de las variables en los tres primeros componentes principales.

\begin{tabular}{lccr}
\hline Variable $^{1}$ & Componente 1 & Componente 2 & Componente 3 \\
\hline $\mathrm{Ar}$ & 0,164264 & 0,321655 & 0,232353 \\
$\mathrm{~L}$ & $-0,367603$ & $-0,00369627$ & $-0,156056$ \\
$\mathrm{Amf}$ & $-0,370027$ & $-0,154629$ & 0,131689 \\
$\mathrm{Af}$ & $-0,388183$ & $-0,114806$ & 0,246172 \\
$\mathrm{Am}$ & $-0,13515$ & $-0,0112169$ & 0,540095 \\
$\mathrm{Ag}$ & 0,266164 & 0,272385 & 0,229606 \\
$\mathrm{Amg}$ & 0,329744 & 0,180752 & 0,17036 \\
$\mathrm{Gf}$ & 0,295049 & $-0,278926$ & 0,227445 \\
$\mathrm{Gm}$ & 0,226259 & $-0,458225$ & $-0,149625$ \\
$\mathrm{Gg}$ & 0,248259 & $-0,434659$ & $-0,119516$ \\
$\mathrm{CH}$ & 0,0713799 & 0,279263 & $-0,454686$ \\
$\mathrm{CC}$ & 0,0922125 & 0,373864 & $-0,227138$ \\
LS & $-0,139641$ & 0,0193087 & $-0,196581$ \\
$\mathrm{MO}$ & 0,269406 & 0,0164166 & 0,236431 \\
$\mathrm{Da}$ & $-0,216523$ & 0,239457 & 0,170279 \\
\hline
\end{tabular}

${ }^{(1)} \mathrm{Ar}$, arcilla; L, limo; Amf, arena muy fina; Af, arena fina; Am, arena media; $\mathrm{Ag}$, arena gruesa; Amg, arena muy gruesa; Gf, gravilla fina; $\mathrm{Gm}$, gravilla media; $\mathrm{Gg}$, gravilla gruesa; $\mathrm{Gv}$, grava; $\mathrm{CH}$, coeficiente higroscópico; $\mathrm{CC}$, capacidad de campo; LS, límite de soltura; MO, materia orgánica; Da, densidad aparente. 

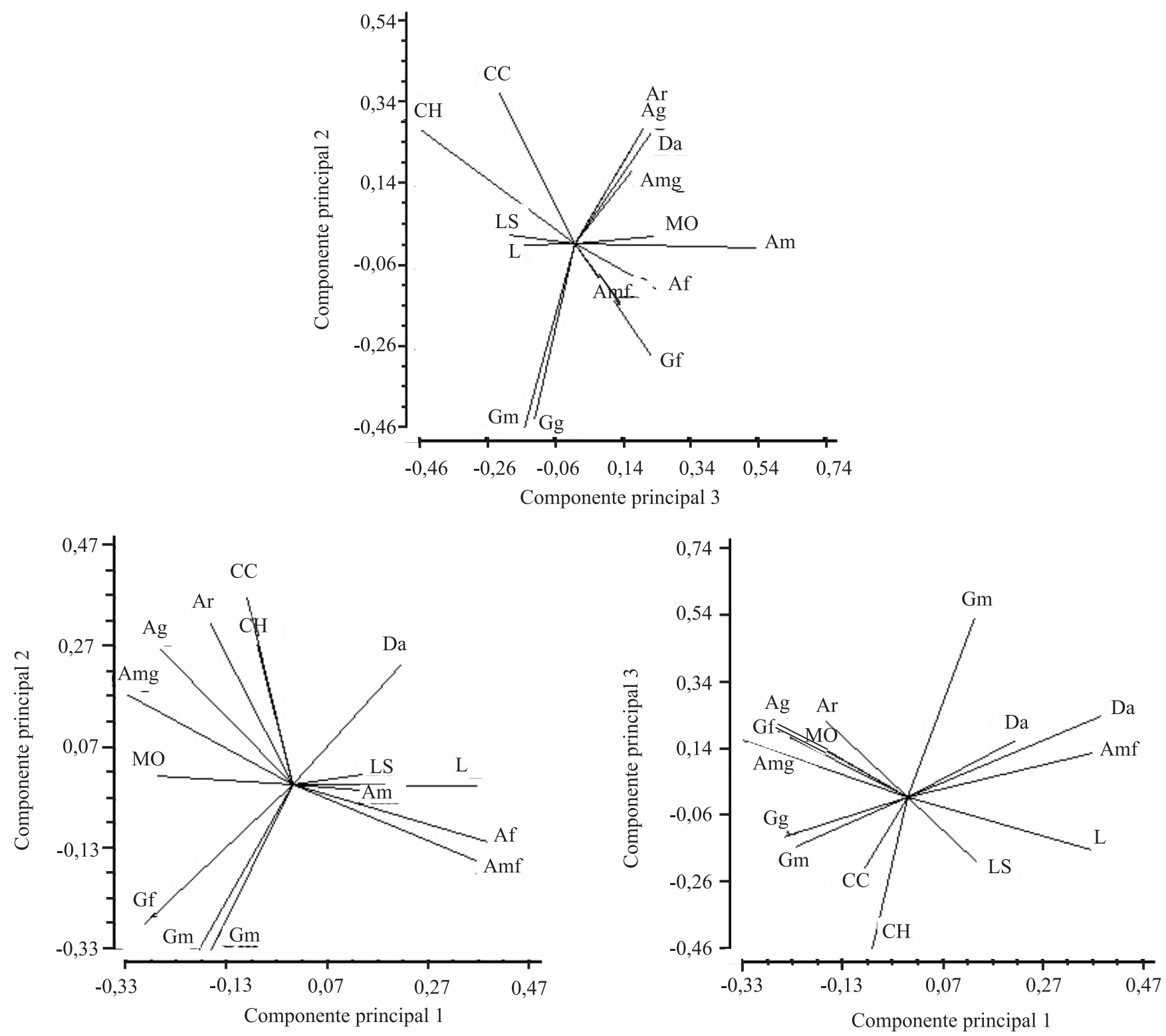

Figura 2. Peso de las variables en los tres primeros componentes principales.

Cuadro 4. Parámetros de la semivariografía de las propiedades estudiadas (Alcance $=63,25 \mathrm{~m}$, lag $=5,89 \mathrm{~m}$, pares en el lag $1=65)$.

\begin{tabular}{|c|c|c|c|c|c|c|}
\hline Variable $^{(1)}$ & Modelo & Nugget & Sill & Rango (m) & $\mathrm{C} / \mathrm{Sill}(\%)^{(2)}$ & $\mathrm{R}^{2}(\%)$ \\
\hline$\overline{\mathrm{CC}}$ & Nugget & 2,701 & 2,701 & - & 0 & 7,8 \\
\hline $\mathrm{Am}$ & Nugget & 0,8793 & 0,8793 & - & 0 & 13,1 \\
\hline $\log \mathrm{Gm}$ & Esférico & 0,0219 & 0,2418 & 27,4 & 90,9 & 66,3 \\
\hline reslogL & Exponencial & 0,0053 & 0,0191 & 90 & 72,3 & 50,5 \\
\hline reslogAf & Gausiano & 0,0013 & 0,0086 & 17,67 & 85,1 & 55,2 \\
\hline $\mathrm{resCH}$ & Exponencial & 0,0116 & 0,1622 & 12,3 & 92,8 & 15,0 \\
\hline
\end{tabular}

$\mathrm{CC}$, capacidad de campo; Am, arena media; Gm, gravilla media; L, limo; Af, arena fina; $\mathrm{CH}$, coeficiente higroscópico; res, residuales; log, logaritmo base 10. ${ }^{(2)}$ Porcentaje del sill que corresponde a variabilidad espacial. 

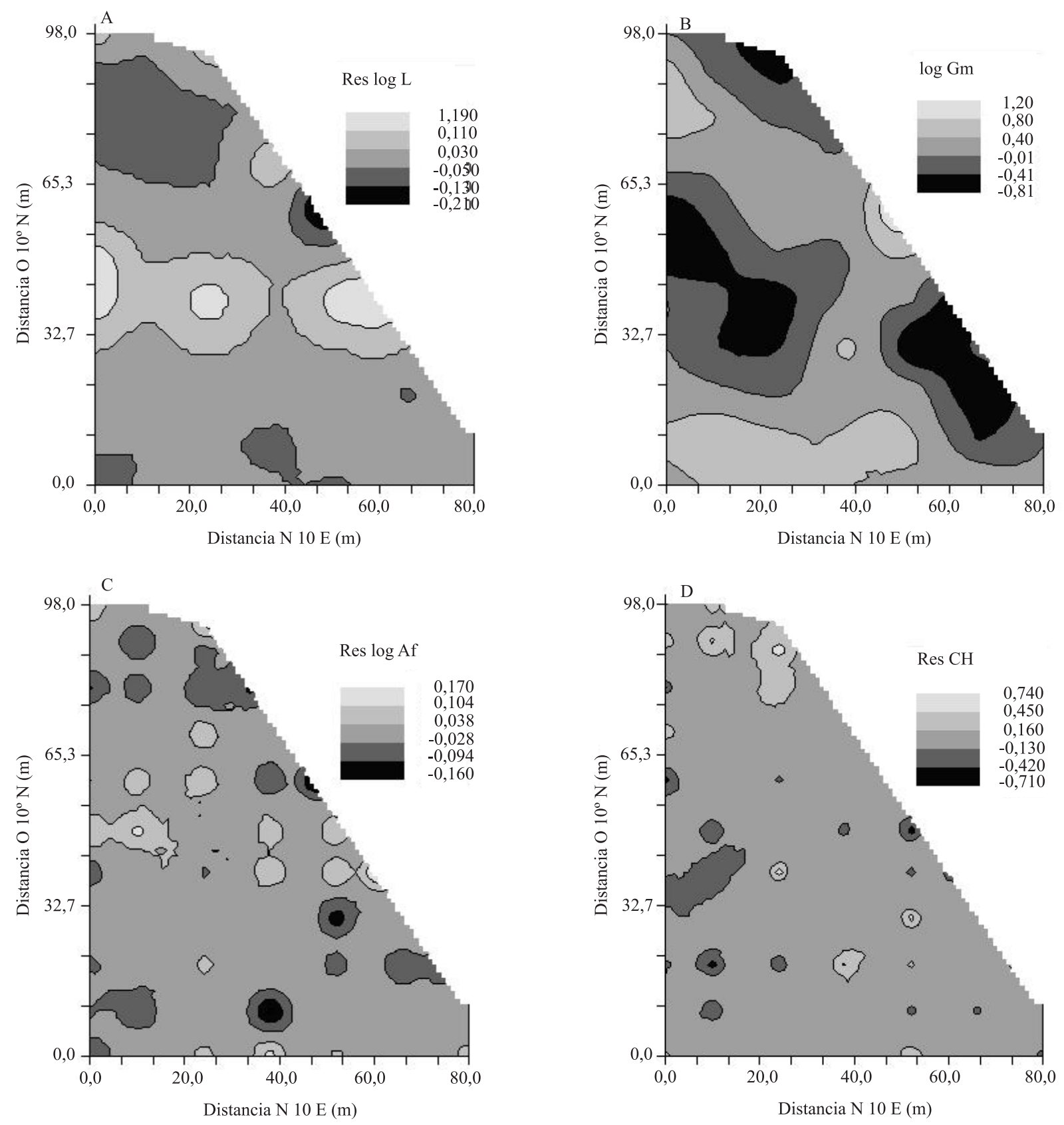

Figura 3. Mapas de la distribución de limo (A), gravilla media (B), arena fina (C), coeficiente higroscópico (D), en el lote experimental.

que tiene su dependencia espacial y poder definir adecuadamente la ubicación de parcelas experimentales en este lote (Lozano P. et al., 2004).

Los mapas de L y Af presentan una tendencia de distribución espacial de estas partículas en franjas amplias localizadas en el sentido $\mathrm{N} 10^{\circ} \mathrm{E}$, es decir, presentando su mayor variabilidad en la dirección $\mathrm{W} 10^{\circ} \mathrm{N}$. Además, presentan una banda de máxima acumulación en el centro del lote. La distribución de la $\mathrm{Gm}$ se presenta en forma diagonal al lote, mientras que el $\mathrm{CH}$ no presenta ningún patrón de distribución.

Los contenidos promedios de $\mathrm{Gm}$ y Af son bajos en el lote experimental $(1,95 \%$ y $5,12 \%$, respectivamente) mientras que el contenido medio de $\mathrm{L}$ es de $37,13 \%$, lo que hace suponer que el limo sea la partícula que probablemente tenga mayor 
impacto en el desgaste de las herramientas en este suelo. Aparte de la cantidad, las gravillas y la arena, por su tamaño, pueden rodar sobre la masa del suelo al ser empujado éste por el implemento con lo que su efecto en el rayado y desgaste de aquel es mínimo. El limo, por ser mucho más pequeño y abundante va a estar más tiempo en contacto con la superficie de la herramienta, rayándola más intensamente y causándole mayor desgaste.

Owsiak (1999) y Natsis et al. (2008) demostraron que en suelos arcillosos la humedad redujo el desgaste de los implementos al reducir la dureza del suelo, mientras que en los suelos de textura liviana no tuvo este efecto, a pesar de reducir también la dureza, comportamiento que relacionaron al tamaño de las partículas. Estas observaciones, y el hecho de que la CC haya estado entre las propiedades seleccionadas en los componentes principales, permiten recomendar que el laboreo en estos suelos se haga con un contenido de humedad muy cercano, por lo menos, al LS, muy similar a la CC, para minimizar el desgaste de los implementos agrícolas.

Teniendo en cuenta la importancia que puede tener el limo en el desgaste de las herramientas, y con base en su comportamiento espacial, se puede recomendar que para estudiar la abrasividad de este suelo las parcelas experimentales se ubiquen a lo largo de la dirección $\mathrm{N} 10^{\circ} \mathrm{E}$ del lote, considerando la franja ubicada entre los 30 y los $60 \mathrm{~m}$ de distancia $\mathrm{W} 10^{\circ} \mathrm{N}$, como una unidad de granulometría diferente al resto del lote por su acumulación de limo. Nótese que en dicha franja también se presentan los contenidos más altos de arena fina y de gravilla media. Esta franja debe ser considerada como diferente al resto del lote y si se utiliza como parte de un mismo experimento se corre el riesgo de que se trabaje con dos poblaciones distintas de suelo.

\section{Conclusiones}

1. De entre las seis variables que explicaron $60 \%$ de la variabilidad física del suelo, los contenidos de limo, arena fina y gravilla media, así como el coeficiente higroscópico presentan alta dependencia espacial y deben ser utilizadas para definir la ubicación de parcelas experimentales que se utilicen para llevar a cabo estudios sobre desgaste de implementos agrícolas en el laboreo del suelo.
2. En el lote experimental se presentan tres sectores de acumulación diferencial de limo y de arena fina, orientados a lo largo de la dirección $\mathrm{N} 10^{\circ} \mathrm{E}$ de la malla de muestreo, presentándose las mayores cantidades de dichas partículas en una banda localizada entre los 30 y $60 \mathrm{~m}$ en la dirección $\mathrm{W} 10^{\circ} \mathrm{N}$.

3. El análisis visual de los mapas de isolíneas de las variables seleccionadas permitió sugerir que las parcelas experimentales se deben ubicar en la dirección $\mathrm{N} 10^{\circ} \mathrm{E}$ de la malla de muestreo.

4. La combinación de los análisis de componentes principales y geoestadístico es muy eficiente en la selección de las propiedades del suelo que más efecto pudieron tener sobre el desgaste de implementos agrícolas y en la determinación del patrón espacial de las mismas.

\section{Agradecimiento}

A la Dirección de Investigaciones de la Universidad Nacional - Sede Medellín por la financiación de los proyectos de investigación. Al Grupo de Tribología y Superficies por la colaboración en aspectos logísticos. Al Laboratorio de Suelos de la Facultad de Ciencias por la determinación de la materia orgánica. Al Centro Agropecuario Cotové de la Facultad de Ciencias Agropecuarias, por la asignación del lote de estudio.

\section{Referencias}

ARANGO, L. Efecto de la variabilidad especial de algunas propiedades físicas y químicas del suelo relacionadas con la producción del forraje del pasto ángleton (Dichanthium aristatum (Poir) C. E. Hubbard). 2004.78p. Tesis (Maestría) Facultad de Ciencias, Medellín.

BHATTI, A.U.; MULLA, D.J.; KOEHLER, F.E.; GURMANI, A.H. Identifying and removing spatial correlation from yield experiments. Soil Science Society of America Journal, v.55, p.1523-1528, 1991.

BOURENNANE, H.; NICOULlAUD, B.; COUTURIER, A.; KING, D. Exploring the spatial relationships between some soil properties and wheat yields in two soil types. Precision Agriculture, v.5, p.521-536, 2004.

CAMBARDELLA, C.A.; MOORMAN, T.B.; NOVAK, J.M.; PARKIN, T.B.; KARLEN, D.L.; TURCO, R.F.; KONOPKA, A.E. Field-scale variability of soil properties in Central Iowa soils. Soil Science Society of America Journal, v.58, p.1501-1511, 1994.

CASTAÑEDA SÁNCHEZ, D.A.; JARAMILLO JARAMILLO, D.F.; COTES TORRES, J.M. Componentes de la variabilidad espacial en el manejo por sitio específico en banano. Pesquisa Agropecuária Brasileira, v.45, p.836-845. 2010. 
CHEN, F.; KISSEL, D.E.; WEST, L.T.; ADKINS, W.; CLARK, R.; RICKMAN, D.; LUVALL, J.C. Field scale mapping of surface soil clay concentration. Precision Agriculture, v.5, p.7-26, 2004.

CORTÉS LOMBADA, A.; MALAGÓN CASTRO, D. Los levantamientos agrológicos y sus aplicaciones múltiples. Bogotá: Fundación Universidad de Bogotá Jorge Tadeo Lozano, 1984. 360p.

DEMEY, J.R.; ADAMS, M.; FREITES, H. Uso del método de análisis de componentes principales para la caracterización de fincas agropecuarias. Agronomía Tropical, v.44, p.475-497, 1994.

DIGGLE, P.J.; RIBEIRO JUNIOR, P.J. Model-based geostatistics. New York: Springer, 2007. p.12-28.

GOOGLE EARTH. Google Earth [home page]. Available at: < http://earth.google.com/>. Accessed on: 20 Oct. 2011.

GUASTAFERRO, F.; CASTRIGNANÒ,A.; DE BENEDETTO, D.; SOLLITTO, D.; TROCCOLI, A.; CAFARELLI, B. A comparison of different algorithms for the delineation of management zones. Precision Agriculture, v.11, p.600-620, 2010.

HENGL, T. A practical guide to geostatistical mapping. $2^{\text {nd }}$ ed. Amsterdam: University of Amsterdam, 2009. 291p.

IQBAL, J.; THOMASSON, J.A.; JENKINS, J.N.; OWENS, P.R.; WHISLER, F.D. Spatial variability analysis of soil physical properties of alluvial soils. Soil Science Society of America Journal, v.69, p.1338-1350, 2005.

JARAMILLO JARAMILLO, D.F. Efecto de la variabilidad sistemática de la producción de fríjol en experimentos de fertilización. Segunda siembra. Revista Facultad Nacional de Agronomía - Medellín, v.59, p.3147-3165, 2006.

JARAMILLO JARAMILLO, D.F. Efecto de la variabilidad sistemática en experimentos de fertilización con fríjol. Primera siembra. Revista Facultad Nacional de Agronomía - Medellín, v.58, p.2717-2732, 2005.

JARAMILLO JARAMILLO, D.F. Variabilidad espacial de las propiedades ándicas de un Andisol hidromórfico del Oriente Antioqueño (Colombia). Revista Facultad Nacional de Agronomía - Medellín, v.62, p.4907-4921, 2009.

JARAMILLO JARAMILLO, D.F.; GONZÁLEZ SÁNCHEZ, H.; ÁLVAREZ MEJÍA, F. Variabilidad espacial de algunas propiedades físico - mecánicas de suelos de textura pesada. CES Medicina Veterinaria y Zootecnia, v.3, p.10-19, 2008.

KRASILNIKOV, P. Variography of discrete soil properties. In: KRASILNIKOV, P.; CARRÉ, F.; MONTANARELLA, L. (Ed.). Soil geography and geostatistics: concepts and applications. Luxembourg: European Commission, 2008. p.12-25.

KRASILNIKOV, P.; SIDOROVA, V. Geostatistical analysis of the spatial structure of acidity and organic carbon in zonal soils of the Russian plain. In: KRASILNIKOV, P.; CARRÉ, F.; MONTANARELLA, L. (Ed.). Soil geography and geostatistics: concepts and applications. Luxembourg: European Commission, 2008. p.55-67.

LOZANO P., Z.; BRAVO, C.; OVALLES, F.; HERNÁNDEZ, R.M.; MORENO, B.; PIÑANGO, L.; GREGORIO VILLANUEVA, J. Selección de un diseño de muestreo en parcelas experimentales a partir del estudio de la variabilidad espacial de los suelos. Bioagro, v.16, p.61-72, 2004.

LOZANO, J.; MADERO, E.; TAFUR, H.; HERRERA, O.; AMÉZQUITA, E. Distribución de un nuevo índice de compactación validado en suelos del Valle del Cauca. Suelos Ecuatoriales, v.34, p.102-111, 2004.

NATSIS, A.; PETROPOULUS, G.; PANDAZARAS, C. Influence of local soil conditions on mouldboard ploughshare abrasive wear. Tribology International, v.41, p.151-157, 2008.

OWSIAK, Z. Wear of spring tine cultivator points in sandy loam and light clay soils in southern Poland. Soil and Tillage Research, v.50, p.333-340, 1999.

PEÑA, R.; RUBIANO, Y.; PEÑA, A.; CHAVES, B. Variabilidad espacial de los atributos de la capa arable de un inceptisol del piedemonte de la cordillera Oriental (Casanare, Colombia). Agronomía Colombiana, v.27, p.111-120, 2009.

SCHABENBERGER, O.; PIERCE, F.J. Contemporary statistical models for the plant and soil sciences. New York: CRC, 2002. 738 p.

SOUZA, Z.M. de; MARQUES JÚNIOR, J.; PEREIRA, G.T.; SÁNCHEZ SÁENZ, C.M. Spatial variability of aggregate stability in Latosols under sugarcane. Revista Brasileira de Ciência do Solo, v.33, p.245-253, 2009.

VAN MEIRVENNE, M. Is the soil variability within the small fields of flanders structured enough to allow precision agriculture? Precision Agriculture, v.4, p.193-201, 2003.

WEBSTER, R.; OLIVER, M.A. Geostatistics for environmental scientists. $2^{\text {nd }}$ ed. Chichester: John Wiley \& Sons, 2007. 315p.

ZAMAN, Q.; SCHUMANN, A.W. Nutrient management zones for citrus based on variation in soil properties and tree performance. Precision Agriculture, v.7, p.45-63, 2006.

ZELEKE, T.B.; SI, B.C. Scaling relationships between saturated hydraulic conductivity and soil physical properties. Soil Science Society of America Journal, v.69, p.1691-1702, 2005.

ZHAO, Y.; PETH, S.; KRÜMMELBEIN, J.; HORN, R.; WANG, Z.; STEFFENS, M.; HOFFMANN, C.; PENG, X. Spatial variability of soil properties affected by grazing intensity in Inner Mongolia grassland. Ecological Modelling, v.205, p.241-254, 2007.

Recebido el 15 de junio de 2011 y aprobado el 31 de octubre del 2011 